\title{
Reduced expression of a-L-Fucosidase-1 (FUCA-1) predicts recurrence and shorter cancer specific survival in luminal $B$ LN+ breast cancer patients
}

\author{
Serena Bonin ${ }^{1, *}$, Alessia Parascandolo ${ }^{2, *}$, Cinzia Aversa ${ }^{1}$, Renzo Barbazza ${ }^{1}$, Nobuo \\ Tsuchida ${ }^{3}$, Maria Domenica Castellone ${ }^{4}$, Giorgio Stanta ${ }^{1}$ and Giancarlo Vecchio ${ }^{5,6,7}$ \\ ${ }^{1}$ Dipartimento di Scienze Mediche, Università di Trieste-Cattinara, Trieste, Italy \\ ${ }^{2}$ IRCSS SDN, Naples, Italy \\ ${ }^{3}$ Graduate School of Medical and Dental Sciences, Tokyo Medical and Dental University, Tokyo, Japan \\ ${ }^{4}$ Istituto di Endocrinologia e Oncologia Sperimentale G.Salvatore, CNR, Naples, Italy \\ ${ }^{5}$ Dipartimento di Medicina Molecolare e Biotecnologie Mediche, Università di Napoli Federico II, Naples, Italy \\ ${ }^{6}$ Istituto Superiore di Oncologia, Naples, Italy \\ ${ }^{7}$ Istituto Superiore di Oncologia, Genoa, Italy \\ *Co-first authors \\ Correspondence to: Giancarlo Vecchio, email: vecchio@unina.it \\ Keywords: breast cancer; luminal B; lymph node metastasis; a-L-Fucosidase-1; immunohistochemistry \\ Received: October 06, 2017 Accepted: February 01, 2018 Epub: February 07, 2018 Published: March 16, 2018 \\ Copyright: Bonin et al. This is an open-access article distributed under the terms of the Creative Commons Attribution License 3.0 \\ (CC BY 3.0), which permits unrestricted use, distribution, and reproduction in any medium, provided the original author and source \\ are credited.
}

\section{ABSTRACT}

Background: The lysosomal enzyme a-L-Fucosidase-1 (FUCA-1) catalyzes the hydrolytic cleavage of terminal fucose residues. FUCA-1 gene is down-regulated in highly aggressive and metastatic human tumors as its inactivation perturbs the fucosylation of proteins involved in cell adhesion, migration and metastases.

Results: Negativity to FUCA-1 was significantly related to the development of later recurrences in breast cancer patients with lymph node involvement at diagnosis. Cancer specific survival of luminal B LN+ patients was influenced by FUCA-1 expression as luminal B LN+ patients with positive expression had a longer cancer specific survival. FUCA-1 mRNA expression was inversely related to cancer stage and lymph node involvement. WB and qPCR analysis of FUCA-1 expression in breast cancer-derived cell lines confirmed an inverse relationship with tumor aggressiveness.

Conclusions: This study shows that, within LN+ breast cancer patients, FUCA-1 is able to identify a sub-set of non recurrent patients characterized by the positive expression of FUCA-1 and that, within luminal B LN+ patients, the expression of FUCA-1 predicts longer cancer specific survival.

Methods: We have analyzed FUCA-1 in 305 breast cancer patients by Immunohistochemistry (IHC), and by QPCR in breast cancer patients and in breast cancer cell lines.

\section{INTRODUCTION}

Breast cancer $(\mathrm{BC})$ is the most frequent female neoplasia representing the first cause of women cancer death (data from http://globocan.iarc.fr/) and a heterogeneous group of tumors both at the molecular and morphological levels. Also the clinical course of the disease is highly variable, at the same tumor grade and stage; some patients are completely cured, while others recur, even 10 years after surgery. In the last decade, mammary carcinomas have been classified molecularly in 5 main subtypes: three estrogen receptor positive (luminal A and B and HER2+ luminal), one HER2+ non luminal and the triple negative (TN). These subtypes have different 
prognosis and clinical course $[1,2]$. Luminal tumors (both $\mathrm{A}$ and $\mathrm{B}$ ) include more than two thirds of all breast cancers [3]. Although they have a better prognosis, patients with luminal tumors may recur and die from the disease even more than 10 years after surgery [4] . Besides the molecular classification, the presence of lymph-nodal metastasis at diagnosis is one of the most relevant prognostic factors for diagnosis of BC patients. Lymph-node negative patients have $75 \%$ survival probability at 20 years of follow-up, while lymph-node positive patients have $40 \%$ survival, similar to TN patients [4]. Therefore, the possibility to detect new biomarkers for a more specific prognosis in the latter group of patients would be relevant.

Previous observations, regarding the role of fucosylation in cancer, indicated that the human lysosomal enzyme $\alpha$-L-fucosidase-1 (FUCA-1, EC number 3.2.1.51) is down-regulated in highly aggressive human tumors such as neuroblastomas [5], breast [6], and colorectal cancers [7]. Since elevated fucose levels are preferentially expressed in metastatic foci versus primary tumors [8-10], it has been suggested that the study of altered fucose in tumor cells could be useful for searching new treatment targets [11]. Yuan et al. [11] hypothesized that a decrease of fucose content might alter the biological behavior of breast cancer cells, and, especially, the interaction among tumor cells, the ECM and endothelial cells, yielding new information for diagnosis and treatment of metastases.

We recently reported [12] that FUCA-1 is expressed at normal levels in less aggressive, differentiated papillary thyroid cancers, whereas it is down-regulated in highly malignant, anaplastic thyroid cancers compared with its expression in normal thyroid tissues. In this work we studied FUCA-1 expression in breast cancer aggressiveness and prognosis with respect to lymph node involvement.

We report here that negativity to FUCA-1 is significantly related to the development of later recurrences in breast cancer patients with lymph node involvement at diagnosis. Furthermore, higher expression of FUCA-1 among luminal B LN+ patients can identify a sub-group of patients with a better outcome.

\section{RESULTS}

\section{Antibody specificity}

The specificity of the FUCA-1 signal detected by IHC was assessed by the pre-absorption test (Figure 1A, 1B). The positive staining pattern was clearly evident in a histological section of luminal breast cancer (Figure 1A). However, as reported in Figure 1B, the staining pattern was completely eliminated after incubation of the antibody with the protein extract of TPC-1 cells expressing high levels of the FUCA-1 protein. As reported in Figure $1 \mathrm{C}$ and $1 \mathrm{D}$, the immunohistochemical staining was positive in normal colon mucosa, and negative in colon adenocarcinoma. Results were considered negative if no staining was detectable or if staining was present in less than $10 \%$ of the cells examined.

\section{Expression levels of FUCA-1 in different histotypes of breast cancers}

Immunohistochemical stainings with the FUCA-1 antibody in normal mammary gland and breast carcinomas with different levels of differentiation are shown in Supplementary Figure 1.

Well-differentiated tumor samples showed a higher number of positive cells for FUCA-1 compared to poorly differentiated tumors.

\section{Patients}

Mean age of patients at diagnosis was 47 years (range 26-55). During the period of observation 18 patients were lost at follow-up because of emigration during follow-up. At diagnosis 154 patients presented axillary lymph node involvement while 151 did not. No significant differences in age at diagnosis were observed between the $\mathrm{LN}-(46.8 \mathrm{y}$, range $32-55 \mathrm{y})$ and $\mathrm{LN}+(47.0 \mathrm{y}$, range $26-55$ y) groups, but the frequency of patients under 35 years was higher in $\mathrm{LN}+$.

The median follow-up time was 16 years (range $0-25)$. In detail, it was 18 years (range $0-25$ ) for the $\mathrm{LN}-$ group and 9 (range $0-24$ ) for the $\mathrm{LN}+$ group ( $p=$ $0.000)$. In the LN- group 45 women (31\%) recurred, while in the $\mathrm{LN}+$ group 98 patients $(67 \%)$ did so. For $4 \mathrm{LN}-$ patients $(3 \%)$ and $11 \mathrm{LN}+$ ones $(7 \%)$ no information about recurrences was available. All clinical and pathological characteristics are reported in Supplementary Table 1.

\section{Relationship between FUCA-1 expression and clinico- pathological features}

The relationship between FUCA-1 expression and clinico-pathological features was evaluated in 204 patients since cores were not always analyzed for technical reasons in the remnants. The results are reported in Figure 2A. The expression of FUCA-1 in BC patients with lymph node positive cancers, considering all molecular subtypes, was negative in $60 \%$ of patients against $40 \%$ being positive, thus suggesting that the lack of expression of FUCA-1 correlates with a more aggressive clinical behaviour of the tumor.

\section{Relationship between FUCA-1 expression, recurrences frequencies and molecular subtypes}

BC primary tumors were classified as follows: 83 luminal A, 77 luminal B, 20 HER-2+, 24 TN subtypes and 1 unclassified. The expression of FUCA-1 was not associated to individual subtypes $(p=0.9)$, except for lymph node positive patients. Considering all molecular 
subtypes of $\mathrm{LN}+$ patients taken together, the lower expression of FUCA-1 was associated to the development of later recurrence (Figure 2B). Negativity to FUCA-1 expression, in fact, was significantly related to the development of later recurrences ( $p=0.03$ ), since $68 \%$ of recurrent patients were negative to FUCA-1, compared to $47 \%$ of non recurrent ones (Figure $2 \mathrm{~B}$ ). This trend was even more evident when only the group of luminal B lymph node positive patients was analysed, $81 \%$ of whom were negative for FUCA-1 expression, as shown in Figure $2 \mathrm{C}(p=0.01)$. This result was not confirmed in lymph node negative patients $(p=0.5)$ (data not shown).

\section{FUCA-1 mRNA expression}

To confirm the relationship between aggressiveness of breast cancer and expression of FUCA-1, we have analyzed by RT-qPCR FUCA-1-specific mRNA expression in the cohort of $\mathrm{LN}+$ and $\mathrm{LN}-$ patients. Figure $3 \mathrm{~A}$ shows that $\mathrm{LN}+$ patients display significantly lower levels of FUCA-1 specific mRNA. Similarly, Figure 3B shows that FUCA-1 mRNA expression is inversely related to $\mathrm{BC}$ tumor stage.

\section{Relationship between FUCA-1 expression and survival}

At the end of follow-up 87 women were alive, 14 died of any cause different from $\mathrm{BC}$, and 90 patients died from breast carcinoma. Thirteen patients were lost at follow-up.

Cancer specific survival of patients was investigated per molecular subtypes with respect to FUCA-1 positivity, as reported in Table 1. Survival of Luminal B patients was influenced by FUCA-1 cytoplasmic staining, as shown in Figure 4A $(p=0.01)$, where a longer cancer specific survival in patients expressing FUCA-1 is evident. After separating the patients' cohort, according to lymph node involvement, luminal $\mathrm{B} \mathrm{LN}+$ patients with a FUCA-1 positive expression had a significantly longer specific survival $(p=0.001)$ (Figure 4C), whereas luminal B LNwomen did not ( $p=0.7$; Figure 4B).

However, considering the entire cohort of patients, without molecular sub-type grouping, there was not significant difference between FUCA-1 expression in LN$(p=0.1)$ and $\mathrm{LN}+$ patients $(p=0.3)$. To better explore the relationship between luminal B patients and FUCA-1
A

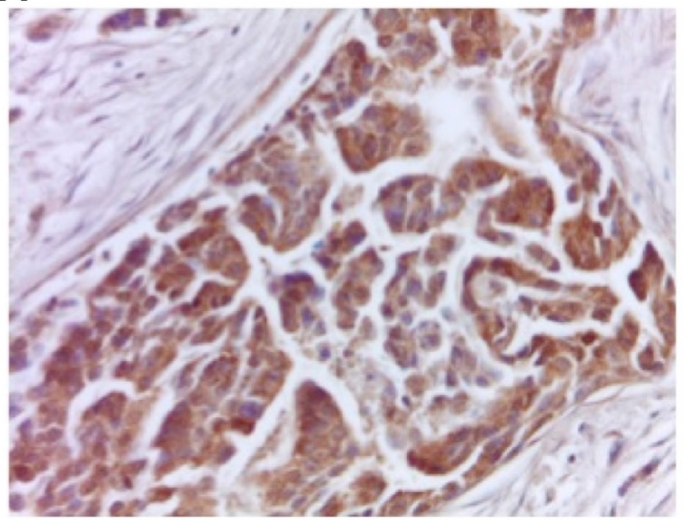

C

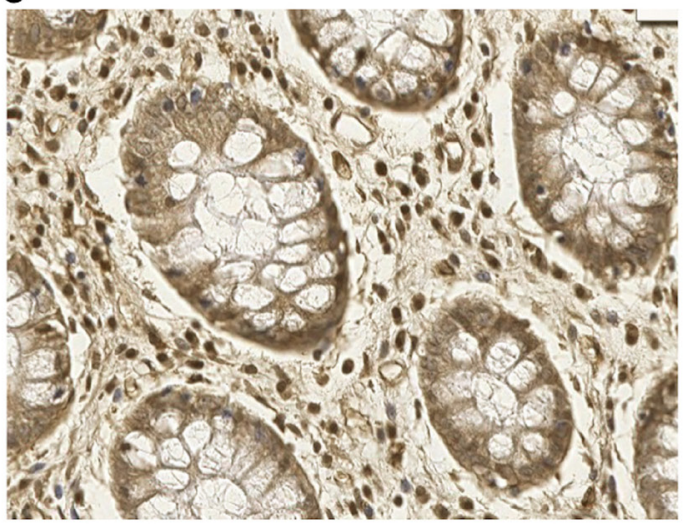

B

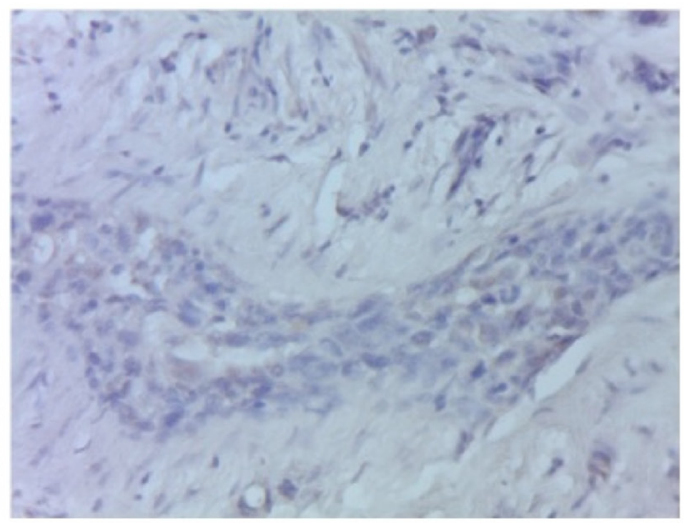

D

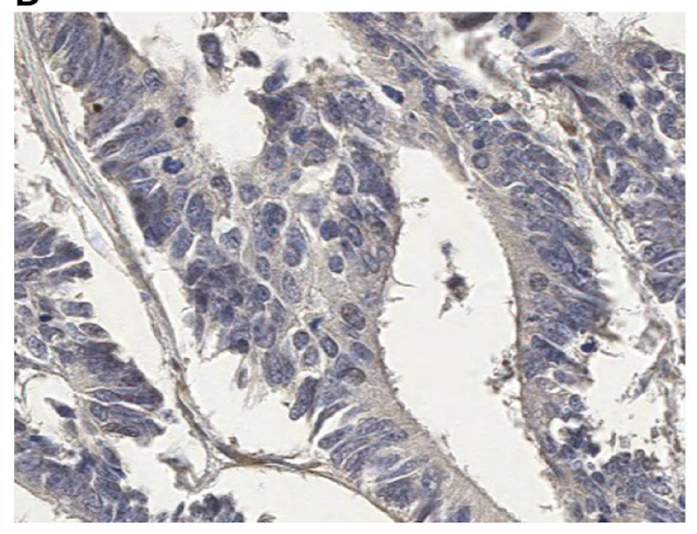

Figure 1: Preabsorption test to verify FUCA-1 polyclonal antibody specificity on luminal A breast cancer lesion. Immunostaining with the FUCA-1 antibody in breast cancer tissue (A) and after preabsorption with a cell extract obtained from a continuous thyroid papillary carcinoma cell line (TPC-1) expressing high levels of the FUCA-1 protein (B). Magnification: 40×; conventional immunohistochemistry performed with di-aminobenzidine as chromogen and hematoxylin as counterstaining. Positive immunostaining on human normal colon mucosa (C) and negative control on colon adenocarcinoma (D) with the same FUCA-1 antibody, Magnification 20×. 
expression, we have investigated the survival curves of lymph node positive patients with respect to the positivity of the surrogate markers that define the luminal B subtype, i.e., positivity to oestrogen and/or progesterone receptor and Ki67 positivity in more than $14 \%$ of cells. Patients survival was influenced by FUCA- 1 expression in LN+ expressing $\mathrm{ER}+, \mathrm{PR}+($ Figure $5 \mathrm{~A}$ and $5 \mathrm{~B})$. The effect of FUCA-1 was even more evident by examining patients with $\mathrm{ER}+, \mathrm{PR}+$ and a Ki67 value higher than $14 \%$ (see Figure 5C and 5D). The same analysis carried out in the LN- subgroup did not show significant differences as reported in the Supplementary Figure 2. Longer cancer specific survival was also observed for Luminal B- HER $2+$ patients expressing FUCA-1 in comparison with those who did not express it (Supplementary Figure 3).

Cox regression analysis for luminal $\mathrm{B} \mathrm{LN}+$ patients using as covariates stage, grade, age at diagnosis, histological type of tumors and FUCA-1 cytoplasmic staining by IHC (Regression $p=0.04$ ) confirmed the protective effect of FUCA-1 $(\mathrm{HR}=0.25,95 \%$ CI $0.09-0.69$ $p=0.008)$ as reported in Supplementary Table 2. Results on the effect of cytoplasmic FUCA-1, Ki67, ER, PR on survival in our cohort of $\mathrm{LN}+\mathrm{BC}$ patients are summarized in Supplementary Table 3, where $p$ values refer to log-
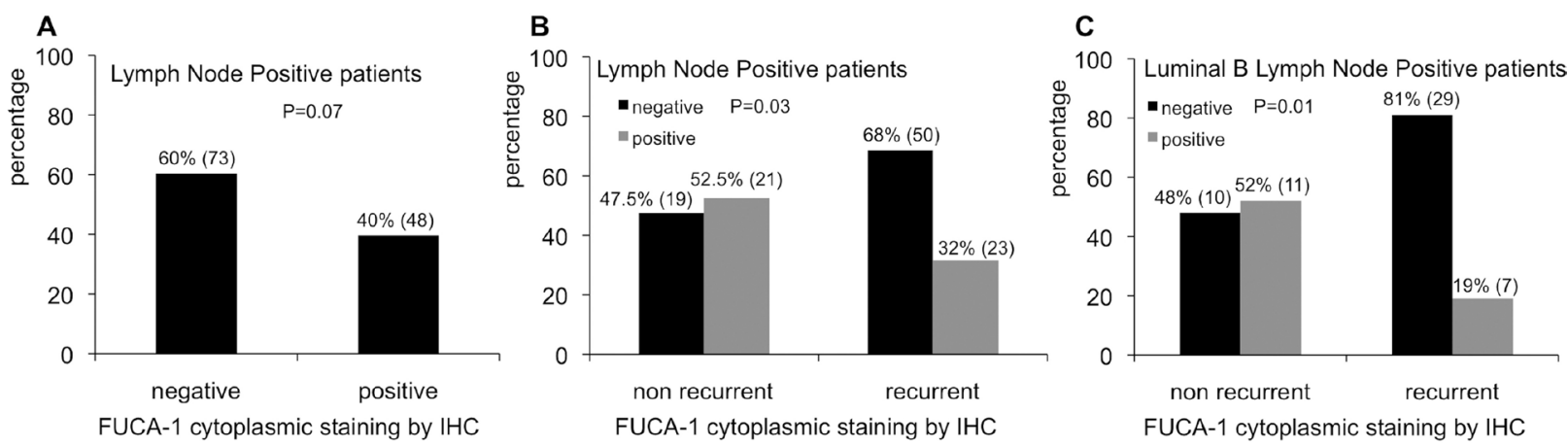

Figure 2: Relative percentages of FUCA-1 immunostaining in LN+ patients (A). Relative percentages of FUCA-1 immunostaining in non recurrent and recurrent $\mathrm{LN}+$ patients $(\mathbf{B})$. Relative percentages of FUCA-1 immunostaining in non recurrent or recurrent luminal B LN+ patients $(\mathbf{C})$.

(A)

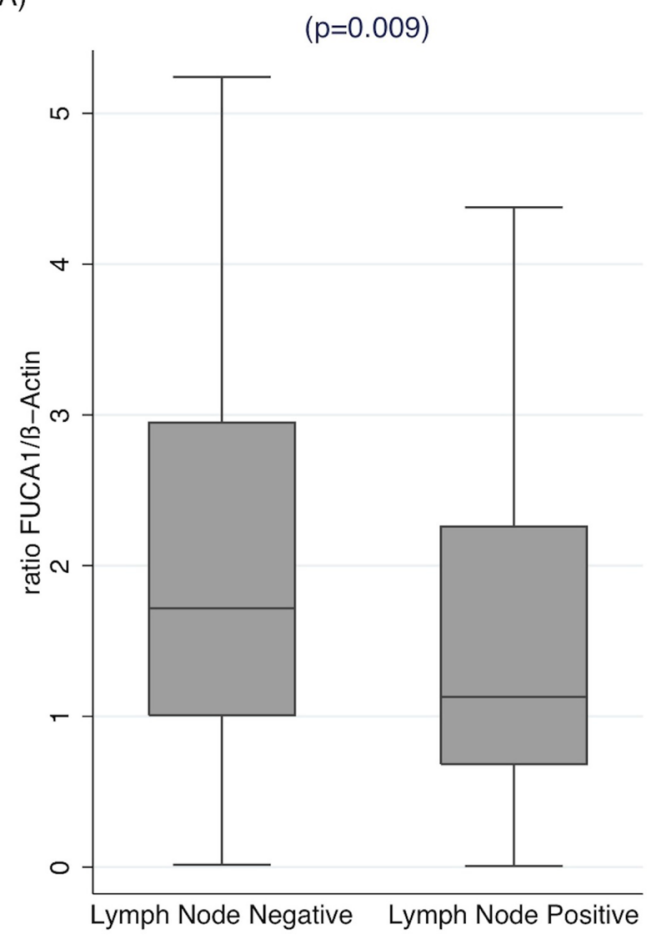

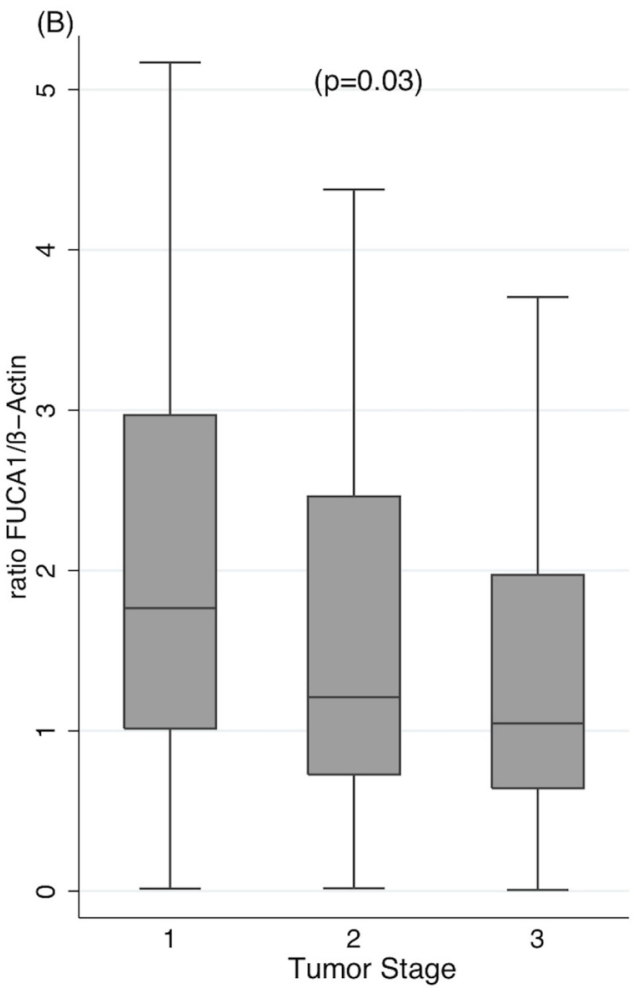

Figure 3: qRT-PCR of FUCA-1-specific RNA extracted from FFPE tissues of the same cohort of patients analyzed in Figures 2, 4 and 5. The relative ratios of FUCA-1 mRNA to $\beta$-actin mRNA is plotted against $\mathrm{LN}+$ or $\mathrm{LN}-$ patients $(\mathbf{A})$ or in patients with different clinical stage (B). 
Table 1: Results of FUCA-1 IHC analysis ( $p$ values refer to Chi2 test and to $t$-test for age at diagnosis)

\begin{tabular}{|c|c|c|c|c|c|}
\hline & & & $\begin{array}{c}\text { FUCA-1 IHC } \\
\text { Negative }\end{array}$ & $\begin{array}{c}\text { FUCA-1 IHC } \\
\text { Positive }\end{array}$ & $p$ \\
\hline \multirow{22}{*}{ 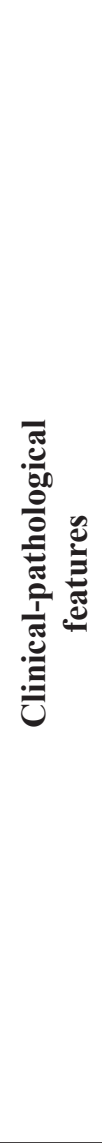 } & Age, mean, years & & 47 & 47.4 & 0.6 \\
\hline & \multirow[t]{3}{*}{ Tumor Stage } & 1 & 39 & 20 & 0.9 \\
\hline & & 2 & 60 & 30 & \\
\hline & & 3 & 34 & 20 & \\
\hline & \multirow[t]{3}{*}{ Tumor grade } & G1 & 13 & 11 & 0.4 \\
\hline & & $\mathrm{G} 2$ & 59 & 33 & \\
\hline & & G3 & 61 & 27 & \\
\hline & \multirow[t]{2}{*}{ Lymph Node Involvement } & No & 60 & 23 & 0.07 \\
\hline & & Yes & 73 & 48 & \\
\hline & \multirow[t]{5}{*}{ Histotype } & Ductal & 106 & 61 & 0.1 \\
\hline & & Lobular & 15 & 3 & \\
\hline & & Medullary & 5 & 0 & $\mathrm{t}$ \\
\hline & & Mucinous & 3 & 4 & \\
\hline & & Tubular & 4 & 3 & \\
\hline & \multirow[t]{2}{*}{ Later recurrences } & No & 52 & 36 & 0.1 \\
\hline & & Yes & 74 & 31 & \\
\hline & \multirow[t]{4}{*}{ Molecular subtypes } & Luminal A & 54 & 29 & 0.9 \\
\hline & & Luminal B & 51 & 24 & \\
\hline & & HER2 & 12 & 8 & \\
\hline & & $\mathrm{TN}$ & 15 & 9 & \\
\hline & \multirow[t]{2}{*}{ Number of Positive LN } & $\leq 3$ & 46 & 31 & 0.7 \\
\hline & & $>3$ & 27 & 13 & \\
\hline
\end{tabular}

rank test obtained for cytoplasmic expression of FUCA-1 in $\mathrm{LN}+\mathrm{BC}$ patients positive for the marked biomarkers.

\section{FUCA-1 protein and mRNA expression in breast cancer cell lines of different molecular subtype}

FUCA-1 protein and mRNA expression was analyzed in a panel of breast cancer cell lines established from patients with different histotypes of $\mathrm{BC}$ tumors (Figure 6). In particular, we analyzed the MCF7 (luminal A), the T47D (luminal A), the BT474 (luminal B), the SKBR3 (HER2+, ER-, PR-) and the MDA-MB231 (claudin low, triple negative) [13] cell lines by WB and qPCR finding that expression of FUCA-1 was strongly decreased in less differentiated molecular subtypes SKBR3 and in the triple negative subtype MDA-MB231. Furthermore, the analysis of a publically available database (Oncomine) (see Supplementary Table 4) shows that a lower expression of FUCA-1 mRNA is characteristic of histologically more invasive and aggressive breast cancer cell lines subtypes.

\section{DISCUSSION}

$\alpha$-L-fucosidases are exoglycosidases widespread in nature [14], ubiquitously expressed in eukaryotic cells. In humans the FUCA-1 gene, coding for the lysosomal enzyme $\alpha$-L-fucosidase-1, has been widely studied [15]. In this study, we investigated the expression of FUCA1 both at mRNA and protein levels in breast cancer (BC). Our results thus showed clearly that negativity to FUCA-1 is significantly related to the development of later recurrences in breast cancer patients with lymph node involvement at diagnosis. Moreover, cancer specific survival of luminal B LN+ patients was influenced by FUCA-1 expression, since these patients, having positive FUCA-1 expression, had a longer survival. Our results thus showed clearly that FUCA-1 is able to define a subgroup of luminal B, lymph node positive patients with a favorable prognosis and that this protein may represent a positive marker to discriminate a subset of $\mathrm{LN}+$ patients who will have a lower risk of recurrence and longer cancer specific survival. 
Other authors have studied the role of FUCA-1 in BC. Milde-Langosh et al. [16] identified in BC FUCA1 among 24 relevant genes, coding for sixteen anabolic and eight catabolic enzymes, with independent prognostic value. Low FUCA-1 expression correlated significantly with a shorter relapse free survival as well as overall survival, while FUCA-1 overexpression was associated with a relatively good outcome, showing an independent prognostic value, in agreement with the present results. Furthermore, FUCA-1 was shown to be up-regulated only in Luminal A breast cancer patients compared to basallike breast cancer patients in a study of glycan-related gene expression profiling in breast cancer subtypes [17]. These authors however did not examine differences of FUCA-1 expression between $\mathrm{LN}+$ and $\mathrm{LN}-$ patients. A decreased expression of the FUCA-1-gene was also found in human colorectal carcinomas, compared to normal mucosa and a gradual decrement in FUCA-1 expression was observed with progression of the disease from earlier to advanced stages [7]. Furthermore, a reduction in the $\alpha$-L-Fucosidase activity in the tumor vs. normal mucosa has been correlated with recurrence in more than $52 \%$ of colorectal carcinoma patients and was considered as a good independent prognostic factor [18].

The role of FUCA-1 in reducing invasiveness in breast and colon cancer could be explained by FUCA-
1 mediated decrease in the composition and quantity of cell surface fucosylation- associated molecules [19]. In line with Cheng et al. (2015) [19], who detected FUCA1 in early stage $\mathrm{BC}$, our findings showed an inverse association between FUCA-1 mRNA and tumor stage. The expression of the FUCA-1 gene has been reported to be directly controlled by p53 [20, 21]. Mutated p53 has been associated with lower expression of FUCA-1 in a set of human thyroid cancer cell lines [21]. In this respect, it is interesting to notice that mutations in p53 represent the most common genetic alteration in breast cancer, found in average $30 \%$ of breast tumors, with higher frequencies in most aggressive cancers (50\% of HER2 amplified BC and $88 \%$ of basal-like carcinomas) whereas its frequency is lower in luminal tumors (17\% of luminal A, $41 \%$ of luminal B) [22].

Thus, it is likely that within luminal B tumors with $\mathrm{LN}+$ higher FUCA-1 expression is associated with the wild type $\mathrm{p} 53$, thereby giving good prognosis.

Furthermore, some markers, such as E-cadherin, CD44 and CD24, which characterize the metastatic potential of human breast cancer cells, have been related to the molecular subtypes. It has been demonstrated that low colony forming activity of human breast cancer cells of luminal subtype is related to increased adhesive properties of these cells, whereas high tumorigenicity of cells of basal
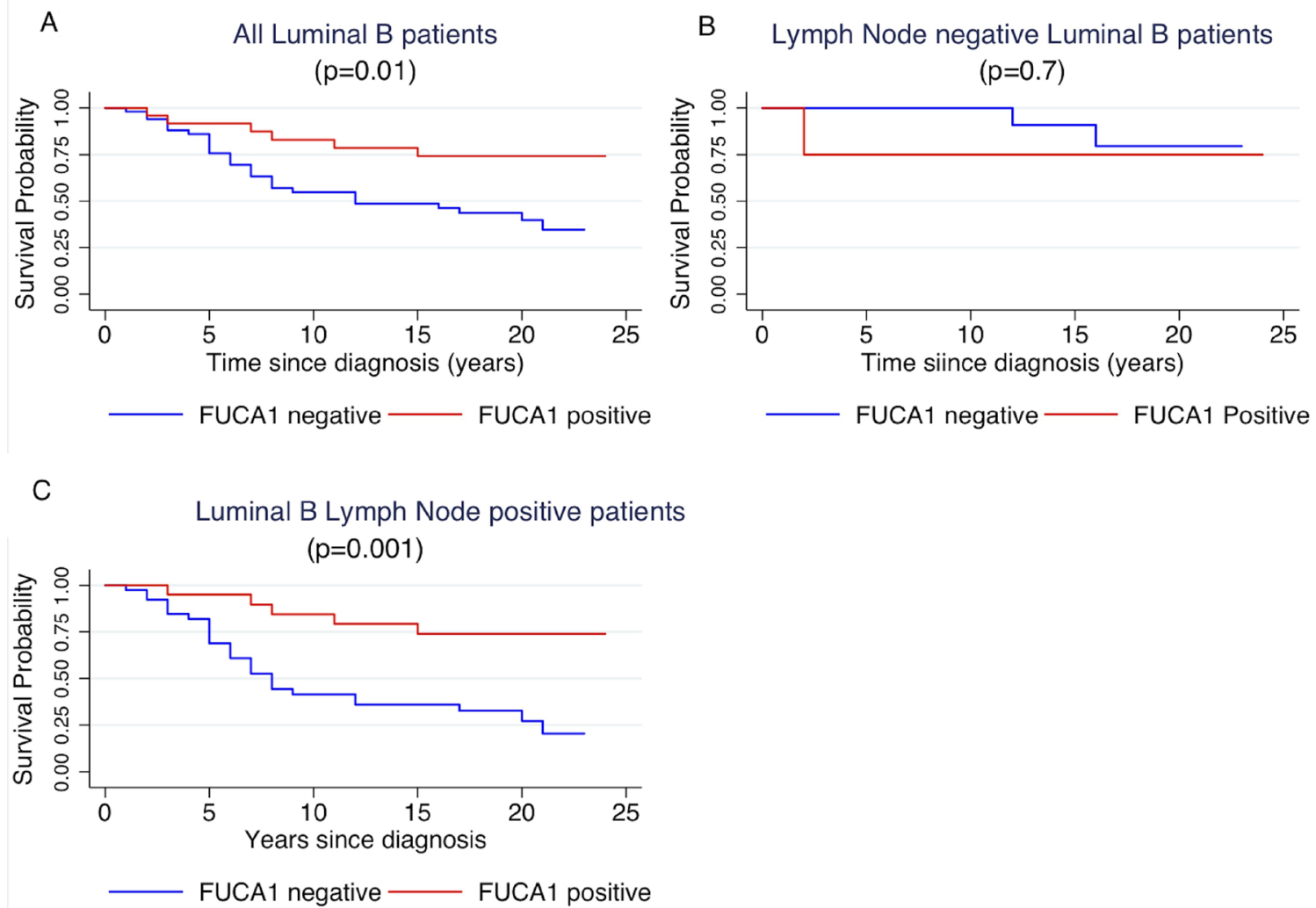

Figure 4: Kaplan- Meier curves of cancer specific survival by positivity or negativity to FUCA-1 immunostaining in all luminal B patients (A), in $\mathrm{LN}$ - luminal B patients (B) and in $\mathrm{LN}+$ luminal B patients $(\mathbf{C})$. 
subtype is connected to weakening of adhesive contacts [23]. These observations are in line with our results on $\mathrm{BC}$ cell lines showing an inverse relationship between FUCA-1 expression and aggressiveness, since claudin low triple-negative (MDA-MB231) and HER2 overexpressing (SKBR3) BC cell lines showed lower expression of FUCA1 in comparison to luminal A ones (MCF-7 and T47D) and luminal B (BT474) both by WB and qPCR.

Our results are also in agreement with those of Ezawa et al. [24], who found that lower FUCA-1 expression was associated with poor prognosis in cancer patients, especially in colorectal and breast cancer patients.

FUCA-1 is a marker of good prognosis in several types of tumors. In the absence of routine gene expression profiling, surrogate IHC markers for molecular breast cancer subtypes represent a more practical means of characterizing $\mathrm{BC}$ tumor types according to prognosis and/or differential response to specific agents [25, 26]. Moreover, the success of new anti-cancer therapies is likely to be dependent upon the use of new biomarkers to detect patients who will benefit from a particular treatment [26]. In this study we have shown that FUCA-1 is able to define a subset of luminal B lymph node positive BC with longer cancer specific and overall survival. Our findings are relevant because most existing biomarkers are predictive or prognostic in lymph node negative BC. Our data clearly show that FUCA-1 may represent
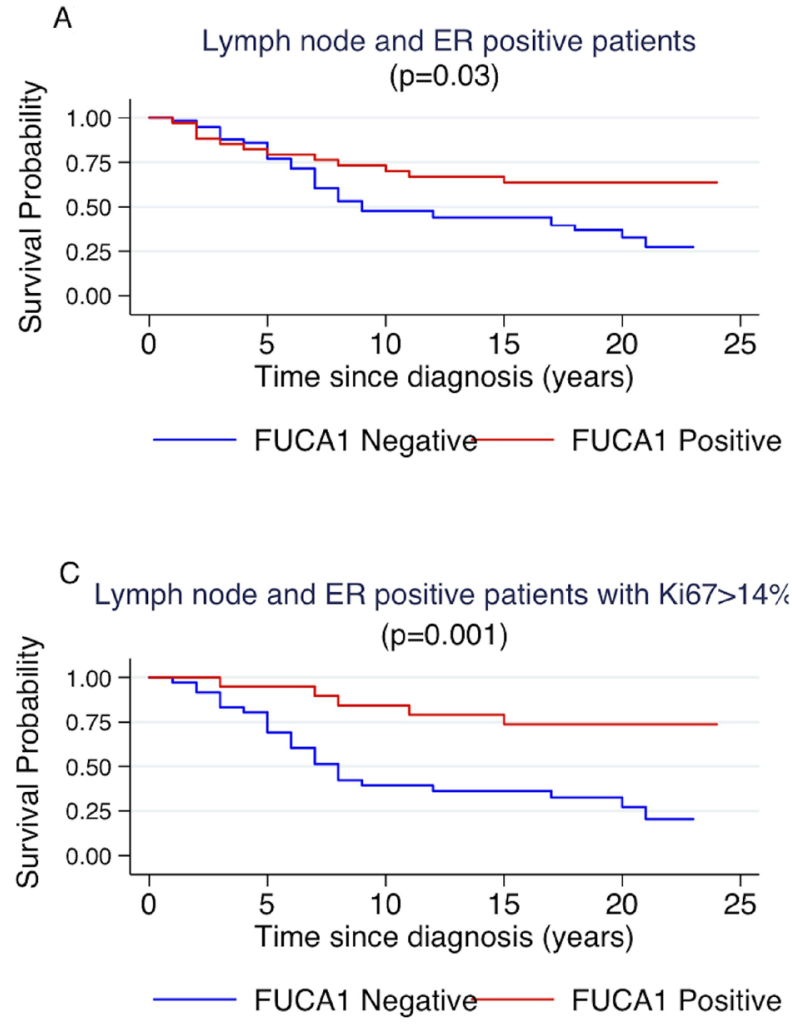

Figure 5: Kaplan-Meier curves of cancer specific survival by positivity or negativity to FUCA-1 immunostaining in LN + ER + patients (A), in $\mathrm{LN}+, \mathrm{PR}+$ patients (B), in $\mathrm{LN}+$, ER+ patients with Ki67 staining higher than $14 \%(\mathbf{C})$ and in $\mathrm{LN}+$, $\mathrm{PR}+$ patients with $\mathrm{Ki67}$ staining higher than $14 \%(D)$.
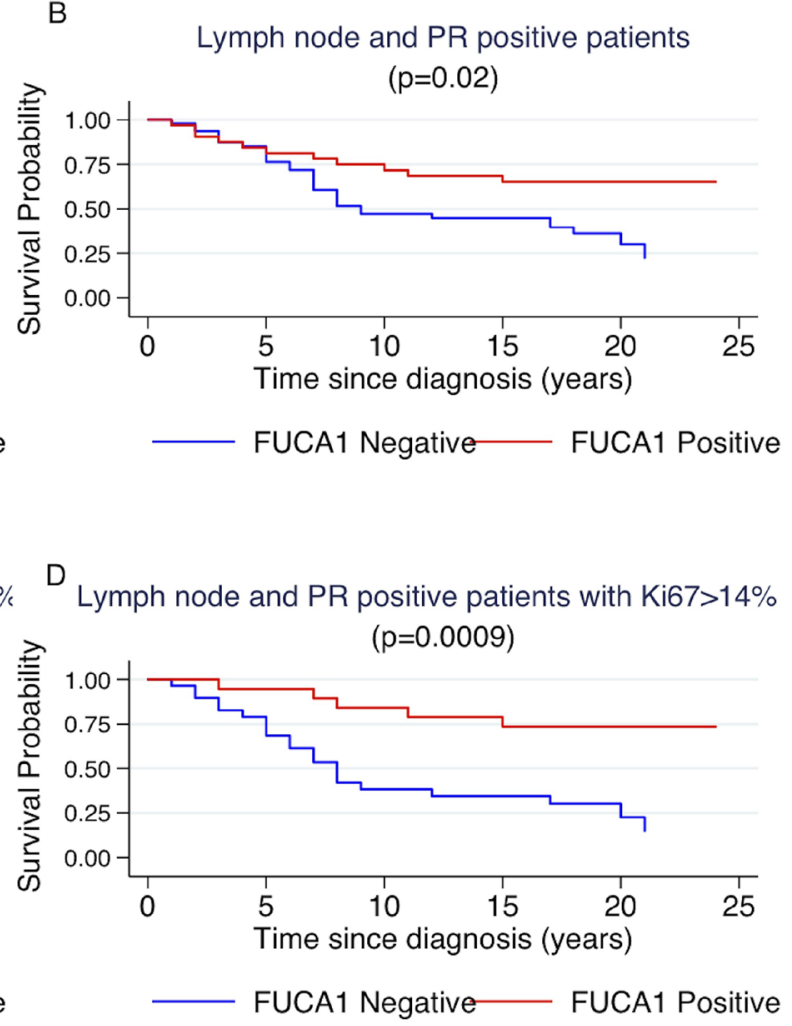

a reliable biomarker for clinical use easily detectable by immunohistochemistry staining. Larger multicenter studies will be necessary to confirm the use of FUCA-1 in clinical practice.

\section{MATERIALS AND METHODS}

\section{Patients}

All patients were resident in a Northern-East province of Italy and were already examined in a previous study [4]. Inclusion criteria were: i) diagnosis of BC at least 15 years before the censoring date of the study (31st December 2008), ii) invasive BC of stage I-III, iii) age at diagnosis 55 years or younger, iv) availability of formalinfixed and paraffin-embedded (FFPE) tissues. Cases with a second primary breast cancer or other malignancies were excluded from the study. Accordingly, 305 patients represented the final cohort for the study, 154 of whom $(50.4 \%)$ had lymph node involvement $(\mathrm{LN}+)$ at diagnosis. FFPE tissue sections of the primary tumor obtained after surgery were used. Clinical information was obtained from medical records. Tumors were reviewed and histologically classified according to the World Health Organization (WHO 2003) [27], graded using Elston and Ellis grading system [28] and grouped into stages according to TNM classification [29]. The patients' cohort was followed for

\section{B}


a maximum of 25 years through the local Cancer Registry from diagnosis of $\mathrm{BC}$ to death or until censoring date. This study was approved by the Ethical Committee of the University of Trieste, as already reported [4].

Patients were treated with mastectomy or breastconserving surgery. All patients submitted to conserving surgery were treated with radiotherapy. All LN+ patients were treated with adjuvant chemotherapy, as already reported [4]. ER-positive patients, both $\mathrm{LN}-$ and $\mathrm{LN}+$, were submitted to tamoxifen therapy. No specific treatment with trastuzumab was performed in HER2positive patients, because this therapy was not available at the time of diagnosis.

\section{Tissue microarray}

Tissue Micro Array (TMA) were built as already described (Pracella et al., 2013) [4]. Briefly, tissues' cores were chosen at the border of the primary tumor. TMA with tissue cores of $1.0 \mathrm{~mm}$ in diameter were built using a tissue-arrayer (Galileo TMA CK3500; Integrated Systems Engineering, Milano, Italy), as previously described [30]. Six TMA blocks containing up to 60 tissue cores each, were obtained. Multiple samples were taken for cases as representative of heterogeneous histological areas and considered positive if at least one core was positive for the specific biomarker. For each TMA block, $4 \mu \mathrm{m}$ thick sections were cut, mounted on Superfrost ${ }^{\circledR}$ Plus (Thermo Scientific) microscope slides and heated at $37^{\circ} \mathrm{C}$ overnight for IHC analysis.

\section{Immunohistochemical staining}

IHC staining was performed following standard procedures [31], according to the manufacturers' instructions for $\alpha$-L-1-Fucosidase (FUCA-1) Polyclonal Antibody (Proteintech, Chicago, USA). Immunostaining was performed manually with the Vectastain Universal Elite ABC kit (Vector Laboratories, Burlingame, CA, USA). Polyclonal antibody diluted 1:50 was applied for 45 minutes after 20 minute antigen retrieval at $\mathrm{pH} 8$ using W-CAP TEC buffer (Bioptica, Milano, Italy) at high temperature, in water bath. For visualization, the DAB Substrate kit for Peroxidase (Vector Laboratories, Burlingame, CA, USA) was used. Molecular subtyping of the entire cohort has been previously performed by IHC

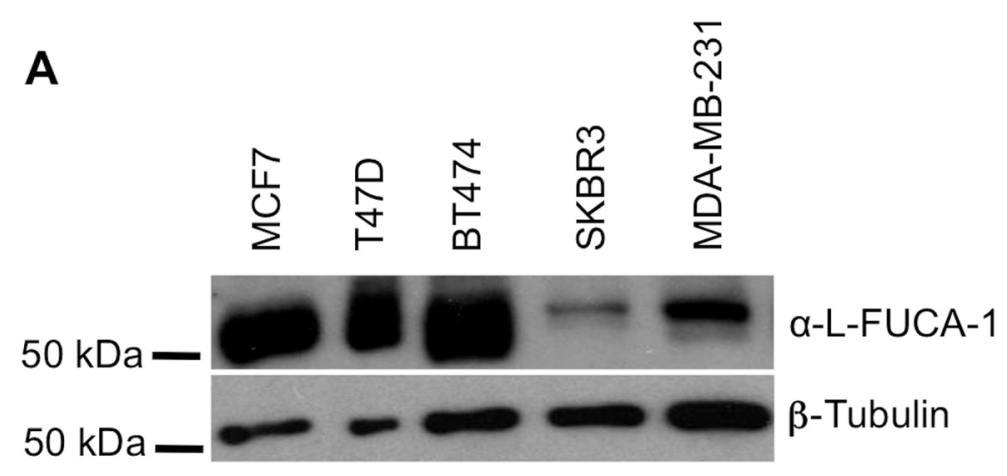

B

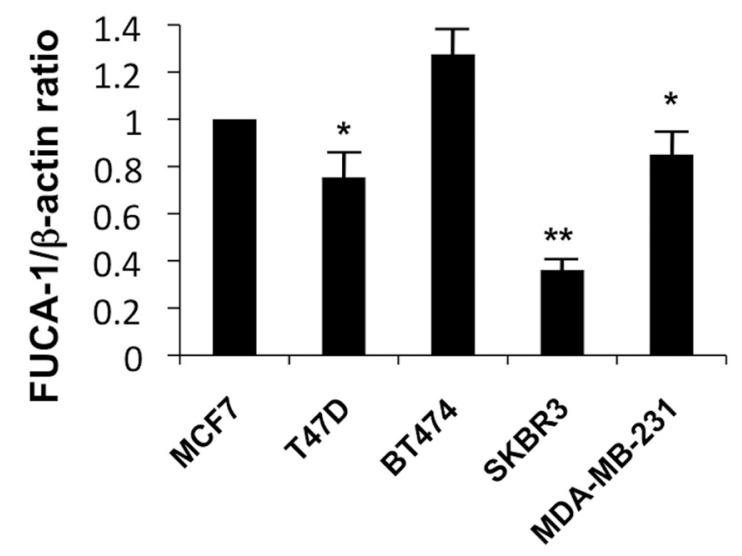

Figure 6: WB and qPCR in breast cancer cell lines extracts. Western blot analysis was performed on a panel of breast cancer cells including MCF7 (luminal A), T47D (Luminal A), BT474 (Luminal B), SKBR3 (HER+), MDA-MB231 (TN, claudin low) using an anti FUCA-1 antibody and normalized by $\beta$ tubulin expression (A). Relative expression of FUCA-1 (FUCA-1/ $\beta$-actin ratio) calculated upon comparison with FUCA-1 expression in MCF7 (luminal A) cells, normalized on beta actin expression is also shown (B). 
using ER, PR , HER2 and Ki67 antibodies as described elsewhere [4].

\section{Evaluation of immunohistochemistry}

Immunostaining was evaluated by two different observers in a blinded fashion (GS, RB), using light microscopy. Cytoplasmic staining was recorded and analyzed. Any positive cytoplasmic staining in tumor cells was considered as positive expression, with a cutoff of $10 \%$ cells. Tumors were classified into four main molecular classes according to the staining profile of the antigen markers as already reported [1,4], notably luminal $\mathrm{A}$ and $\mathrm{B}, \mathrm{Her} 2$ overexpressing, and (TN). If the tumors exhibited markers staining that did not meet the above-mentioned panel criteria, they were defined as 'unclassified'.

\section{RT-PCR assay}

FUCA-1 gene expression was quantitatively measured by Real Time-PCR (qRT-PCR). Briefly, total RNA was extracted from FFPE tissues after TMA microdissection, as previously described [32]. For each sample $4 \mu \mathrm{g}$ of total RNA were treated with DNase, as described [33]. Complementary DNA synthesis was performed from 1.2 $\mu \mathrm{g}$ RNA, using Moloney Murine Leukemia Virus (M-MLV) reverse transcriptase (Invitrogen, Karlsruhe, Germany) by random hexamers priming in a final volume of $20 \mu \mathrm{l}$, as described elsewhere [34]. Expression levels of FUCA-1 and $\beta$-Actin genes were analyzed by real-time PCR using a Mastercycler ${ }^{\circledR}$ ep Realplex (Eppendorf, Hamburg, Germany). PCR assays were performed in duplicates using the JumpStart ${ }^{\mathrm{TM}}$ Taq ReadyMix ${ }^{\mathrm{TM}}$ for Quantitative PCR (Sigma-Aldrich, St. Louis, MO, USA) according to the manufacturer's instructions. In each run, $30 \mathrm{ng}$ of cDNA for $\beta$-Actin and $135 \mathrm{ng}$ for FUCA-1 were amplified in a final volume of $20 \mu \mathrm{l}$. Cycle conditions are reported in Supplementary Table 5. To exclude contamination, negative controls without cDNA were included in every run. RNA extracted from the breast cancer cell line MCF-7 was used as positive control. Primer and probe sequences, created by the use of the Primer Express software (Applied Biosystem, Darmstadt, Germany) and Primer3 (http:// primer3plus.com/primer3web/primer3web input.htm), are reported in Supplementary Table 5. Gene expression levels were normalized using $\beta$-Actin as reference gene, while a mixture of six samples of the cohort were pooled and used as calibrator. The relative quantitation was determined using the method proposed by Pfaffl [35]. For FUCA-1 determination in cultured cell lines, total RNA was isolated with the RNeasy Kit (Qiagen, Crawley, West Sussex, UK). One $\mu \mathrm{g}$ of RNA from each sample was reverse-transcribed with the QuantiTect ${ }^{\circledR}$ Reverse Transcription kit (Qiagen). PCR reactions were performed in triplicates and fold changes were calculated with the formula: 2- (sample 1 $\Delta \mathrm{Ct}$ - sample $2 \Delta \mathrm{Ct}$ ), where $\Delta \mathrm{Ct}$ is the difference between the amplification fluorescent thresholds of the mRNA of interest and the mRNA of $\beta$-Actin used as an internal reference.

\section{Cultured cell lines}

Breast adenocarcinoma luminal A cells (MCF7 and T47D), luminal B (BT474), HER2+ (SK-BR3) and TN (MDA-MB231) cells were obtained from Dr. Aniello Cerrato (IEOS/CNR) and Prof. Roberto Bianco (University of Naples Federico II). TPC1 cells, derived from a papillary thyroid carcinoma, were obtained by $\mathrm{M}$. Nagao (Carcinogenesis Division, National Cancer Center Research Institute, Tokyo, Japan). Cells were grown in Dubecco's modified MEM (DMEM) or RPMI1640 medium supplemented with $10 \%$ fetal bovine serum (FBS). Media were supplemented with $2 \mathrm{mM} \mathrm{L-glutamine}$ and 100 units/ml penicillin-streptomycin (GIBCO).

\section{Antibodies}

Anti -FUCA-1 is a polyclonal antibody from Proteintech (16420-1-AP) which recognizes the $\alpha$-L-1Fucosidase protein. Monoclonal anti- $\beta$-tubulin (\#T9026) antibody was from Sigma Aldrich (St Louis, MO, USA). Secondary antibodies coupled to horseradish peroxidase were from Amersham Pharmacia Biotech (Piscataway, NJ, USA).

\section{Immunoblotting}

Protein lysates were prepared according to standard procedures. Briefly, cells were harvested in lysis buffer (50 mM Hepes, pH 7.5, $150 \mathrm{mM} \mathrm{NaCl}, 10 \%$ glycerol, $1 \%$ Triton X-100, 1 mM EGTA, $1.5 \mathrm{mM} \mathrm{MgCl}_{2}, 10 \mathrm{mM}$ $\mathrm{NaF}, 10 \mathrm{mM}$ sodium pyrophosphate, $1 \mathrm{mM} \mathrm{Na} 3 \mathrm{VO}_{4}$, $10 \mu \mathrm{g}$ of aprotinin $/ \mathrm{ml}, 10 \mu \mathrm{g}$ of leupeptin $/ \mathrm{ml}$ ) and clarified by centrifugation at $10,000 \times g$. Protein concentration was estimated with a modified Bradford assay (Bio-Rad) and lysates were submitted to Western blot. Membranes were probed with the above mentioned antibodies. Immune complexes were revealed by an enhanced chemiluminescence detection kit (ECL, Amersham Pharmacia Biotech). Signal intensity was quantitated using a Phosphorimager (Typhoon 8600, Amersham Pharmacia Biotech) interfaced with the Image Quant software.

\section{Statistical analysis}

Associations between clinical-pathological factors and categories of markers were tested for significance using the chi-square test (or Fisher's exact test, depending on sample size) for categorical variables. Cancer specific survival (CSS), defined as the lapse of time between the date of diagnosis and the date of $\mathrm{BC}$ specific death or the end of follow-up (FU) was the end point evaluated 
in this study. The log-rank test and Kaplan-Meyer curves were used to check the dependence of patients' survival on single variables. To estimate the joint effects of the analysed covariates on patients' survival and confirm the results of the log-rank test, data were analyzed by fitting Cox proportional hazard regression model. Cox analysis included pathological variables (histologic type of tumor, tumor grade, tumor size, number of positive lymph nodes and age at diagnosis) and molecular markers which resulted previously significant at log-rank test. All $p$-values are two-sided with values $<0.05$ regarded as statistically significant. $P$-values between 0.05 and 0.07 were considered as "borderline". Statistical analyses were performed with the Stata/SE 12 package (Stata, College Station, TX).

\section{ACKNOWLEDGMENTS}

This work was partially supported by Progetto PON01_02782 "Novel nanotech strategies for development of drugs and diagnostics for targeting of circulating cancer cells" of the MIUR, Italy to the Istituto Superiore di Oncologia (ISO) and by the project "Role of $\alpha$-L-fucosidase -1 in thyroid and breast cancer" awarded by the Istituto Banco di Napoli -Fondazione, Naples Italy.

Alessia Parascandolo was the recipient of a post-doctoral fellowship by Istituto Banco di NapoliFondazione, Naples Italy.

The Authors are grateful to Prof. Massimo Santoro for his continuous advice and for critically revising the manuscript and to Dr. Anna Maria Cirafici and Gennaro Cito for excellent technical assistance.

\section{CONFLICTS OF INTEREST}

None.

\section{REFERENCES}

1. Sorlie T, Perou CM, Tibshirani R, Aas T, Geisler S, Johnsen H, Hastie T, Eisen MB, van de Rijn M, Jeffrey SS, Thorsen T, Quist H, Matese JC, et al. Gene expression patterns of breast carcinomas distinguish tumor subclasses with clinical implications. Proc Natl Acad Sci U S A. 2001; 98:10869-10874.

2. Weigelt B, Baehner FL, Reis-Filho JS. The contribution of gene expression profiling to breast cancer classification, prognostication and prediction: a retrospective of the last decade. J Pathol. 2010; 220:263-280.

3. Cheang MC, Chia SK, Voduc D, Gao D, Leung S, Snider J, Watson M, Davies S, Bernard PS, Parker JS, Perou CM, Ellis MJ, Nielsen TO. Ki67 index, HER2 status, and prognosis of patients with luminal B breast cancer. J Natl Cancer Inst. 2009; 101:736-750.
4. Pracella D, Bonin S, Barbazza R, Sapino A, Castellano I, Sulfaro S, Stanta G. Are breast cancer molecular classes predictive of survival in patients with long follow-up? Dis Markers. 2013; 35:595-605.

5. Krause A, Combaret V, Iacono I, Lacroix B, Compagnon C, Bergeron C, Valsesia-Wittmann S, Leissner P, Mougin B, Puisieux A. Genome-wide analysis of gene expression in neuroblastomas detected by mass screening. Cancer Lett. 2005; 225:111-120.

6. Daves MH, Hilsenbeck SG, Lau CC, Man TK. Metaanalysis of multiple microarray datasets reveals a common gene signature of metastasis in solid tumors. BMC Med Genomics. 2011; 4:56.

7. Otero-Estevez O, Martinez-Fernandez M, Vazquez-Iglesias L, Paez de la Cadena M, Rodriguez-Berrocal FJ, MartinezZorzano VS. Decreased expression of alpha-L-fucosidase gene FUCA1 in human colorectal tumors. Int J Mol Sci. 2013; 14:16986-16998.

8. Fernandez-Rodriguez J, Paez de la Cadena M, MartinezZorzano VS, Rodriguez-Berrocal FJ. Fucose levels in sera and in tumours of colorectal adenocarcinoma patients. Cancer Lett. 1997; 121:147-153.

9. Huang W, Li X. [Significance of fucose expression in lung carcinoma and their brain metastases]. [Article in Chinese]. Zhonghua Bing Li Xue Za Zhi. 2000; 29:259-262.

10. Listinsky JJ, Listinsky CM, Alapati V, Siegal GP. Cell surface fucose ablation as a therapeutic strategy for malignant neoplasms. Adv Anat Pathol. 2001; 8:330-337.

11. Yuan K, Kucik D, Singh RK, Listinsky CM, Listinsky JJ, Siegal GP. Alterations in human breast cancer adhesionmotility in response to changes in cell surface glycoproteins displaying alpha-L-fucose moieties. Int J Oncol. 2008; 32:797-807.

12. Vecchio G, Parascandolo A, Allocca C, Ugolini C, Basolo F, Moracci M, Strazzulli A, Cobucci-Ponzano B, Laukkanen MO, Castellone MD, Tsuchida N. Human $\alpha$-L-fucosidase-1 attenuates the invasive properties of thyroid cancer. Oncotarget. 2017; 8:27075-27092. https://doi.org/10.18632/ oncotarget.15635.

13. Holliday DL, Speirs V. Choosing the right cell line for breast cancer research. Breast Cancer Res. 2011; 13:215.

14. Intra J, Perotti ME, Pavesi G, Horner D. Comparative and phylogenetic analysis of alpha-L-fucosidase genes. Gene. 2007; 392:34-46.

15. Willems PJ, Seo HC, Coucke P, Tonlorenzi R, O'Brien JS. Spectrum of mutations in fucosidosis. Eur J Hum Genet. 1999; 7:60-67.

16. Milde-Langosch K, Karn T, Schmidt M, zu Eulenburg C, Oliveira-Ferrer L, Wirtz RM, Schumacher U, Witzel I, Schutze D, Muller V. Prognostic relevance of glycosylationassociated genes in breast cancer. Breast Cancer Res Treat. 2014; 145:295-305.

17. Potapenko IO, Luders T, Russnes HG, Helland A, Sorlie T, Kristensen VN, Nord S, Lingjaerde OC, Borresen-Dale AL, 
Haakensen VD. Glycan-related gene expression signatures in breast cancer subtypes; relation to survival. Mol Oncol. 2015; 9:861-876.

18. Fernandez-Rodriguez J, Ayude D, de la Cadena MP, Martinez-Zorzano VS, de Carlos A, Caride-Castro A, de Castro G, Rodriguez-Berrocal FJ. Alpha-L-fucosidase enzyme in the prediction of colorectal cancer patients at high risk of tumor recurrence. Cancer Detect Prev. 2000; 24:143-149.

19. Cheng TC, Tu SH, Chen LC, Chen MY, Chen WY, Lin YK, Ho CT, Lin SY, Wu CH, Ho YS. Down-regulation of alpha-L-fucosidase 1 expression confers inferior survival for triple-negative breast cancer patients by modulating the glycosylation status of the tumor cell surface. Oncotarget. 2015; 6:21283-21300. https://doi.org/10.18632/oncotarget. 4238.

20. Baudot AD, Crighton D, O'Prey J, Somers J, Sierra Gonzalez P, Ryan KM. p53 directly regulates the glycosidase FUCA1 to promote chemotherapy-induced cell death. Cell Cycle. 2016; 15:2299-2308.

21. Tsuchida N, Ikeda MA, Ishino Y, Grieco M, Vecchio G. FUCA1 is induced by wild-type p53 and expressed at different levels in thyroid cancers depending on p53 status. Int J Oncol. 2017; 50:2043-2048.

22. Bertheau P, Lehmann-Che J, Varna M, Dumay A, Poirot B, Porcher R, Turpin E, Plassa LF, de Roquancourt A, Bourstyn E, de Cremoux P, Janin A, Giacchetti S, et al. p53 in breast cancer subtypes and new insights into response to chemotherapy. Breast. 2013; 22:S27-29.

23. Chekhun S, Bezdenezhnykh N, Shvets J, Lukianova N. Expression of biomarkers related to cell adhesion, metastasis and invasion of breast cancer cell lines of different molecular subtype. Exp Oncol. 2013; 35:174-179.

24. Ezawa I, Sawai Y, Kawase T, Okabe A, Tsutsumi S, Ichikawa H, Kobayashi Y, Tashiro F, Namiki H, Kondo T, Semba K, Aburatani H, Taya Y, et al. Novel p53 target gene FUCA1 encodes a fucosidase and regulates growth and survival of cancer cells. Cancer Sci. 2016; 107:734-745.

25. Hudis CA, Gianni L. Triple-negative breast cancer: an unmet medical need. Oncologist. 2011; 16:1-11.

26. Penault-Llorca F, Viale G. Pathological and molecular diagnosis of triple-negative breast cancer: a clinical perspective. Ann Oncol. 2012; 23:vi19-22.
27. Ellis IO, Schnit SJ, Sastre-Garau X, Bussolati G, Tavassoli FA, Eusebi V, Peterse JL, Mukai K, Tabar L, Jacquemier J, Cornelisse CJ, Sasco AJ, Kaaks R, et al. Invasive Breast Carcinoma. In: Tavassoli FA, Devilee P, eds. Pathology and Genetics of Tumours of the Breast and Female Genital Organs. (Lyon: IARC Press). 2003; 13-59.

28. Elston CW, Ellis IO. Pathological prognostic factors in breast cancer. I. The value of histological grade in breast cancer: experience from a large study with long-term follow-up. Histopathology. 1991; 19:403-410.

29. Edge S, Byrd DR, Compton CC, Fritz AG, Greene FL, Trotti A. AJCC Cancer Staging Manual. (New York: Springer-Verlag ). 2010.

30. Faoro V, Sapino A. Tissue Microarray (TMA). In: Stanta G, ed. Guidelines for Molecular Analysis in Archive Tissues. (Berlin, Heidelberg: Springer Berlin Heidelberg). 2011; 23-26.

31. Goldstein NS, Hewitt SM, Taylor CR, Yaziji H, Hicks DG; Members of Ad-Hoc Committee On Immunohistochemistry Standardization. Recommendations for improved standardization of immunohistochemistry. Appl Immunohistochem Mol Morphol. 2007; 15:124-133.

32. Bonin S, Pascolo L, Croce LS, Stanta G, Tiribelli C. Gene expression of $\mathrm{ABC}$ proteins in hepatocellular carcinoma, perineoplastic tissue, and liver diseases. Mol Med. 2002; 8:318-325.

33. Dotti I, Bonin S. DNase Treatment of RNA. In: Stanta G, ed. Guidelines for Molecular Analysis in Archive Tissues. (Berlin, Heidelberg: Springer Berlin Heidelberg). 2011; 87-90.

34. Nardon E, Donada M, Bonin S, Dotti I, Stanta G. Higher random oligo concentration improves reverse transcription yield of cDNA from bioptic tissues and quantitative RT-PCR reliability. Exp Mol Pathol. 2009; 87:146-151.

35. Pfaffl MW. A new mathematical model for relative quantification in real-time RT-PCR. Nucleic Acids Res. 2001; 29:e45. 